\title{
A Compact and Easy to Fabricate E-plane Waveguide Bend
}

\author{
John D. Garrett, Alexander W. Pollak, Ghassan Yassin, and Manju Henry
}

\begin{abstract}
In this paper, we present a new E-plane rectangular waveguide bend that can be micro-machined using split-block fabrication. The $90^{\circ}$ bend is created by machining two linear channels with a notch left in the outside corner. This notch significantly improves the return loss and the insertion loss of the bend. The primary advantage of this technique is that it produces a compact waveguide bend, minimizing both the size and the conduction loss of the waveguide circuit. Another advantage is that this technique only requires linear channels to be machined, thus it can be fabricated without requiring a computer numerically controlled (CNC) milling machine. The performance of this waveguide bend was optimized using electromagnetic simulation software and the optimal reflection coefficient was found to be below $-28 \mathrm{~dB}$ across the entire operational bandwidth of the waveguide. The simulated design was then validated using two experimental prototypes that were tested at microwave (12-18 GHz) and millimeter frequencies (140-220 GHz).
\end{abstract}

\section{INTRODUCTION}

W AVEGUIDE bends require careful fabrication to prevent signal leakage and attenuation. This is especially true at millimeter wavelengths because the conduction losses become much higher and the waveguide dimensions become much smaller. At these frequencies, waveguide components are often created through the use of split-block fabrication, which involves machining the waveguide in two separate blocks and then combining these blocks to form the hollow tube of the waveguide. Split-block fabrication has been used to create a variety of waveguide components including bandpass filters [1], power dividers [2] and directional couplers [3].

The division in split-block waveguides is normally in the E-plane to avoid disrupting the surface currents. Therefore, to create a standard rectangular waveguide, with inner dimensions $a \times b$ where $a=2 \cdot b$, split-block fabrication requires two channels, each with inner dimensions $b \times b$. For waveguides at millimeter wavelengths, the cutting tools that are used to create these channels often have diameters equal to the waveguide's height $b$. This helps to reduce the amount of bending in the cutting tool, but it means that it is impossible to create optimal waveguide bends with mitered or rounded corners (Fig. 1(a) and 1(b), respectively), and only non-optimal rounded bends are possible (Fig. 1(c)). Alternatively, large sweeping arcs can be created with radii $\sim 2 \lambda_{g}$ where $\lambda_{g}$ is the guided wavelength (Fig. 1(d)); however, they require

J.D. Garrett, A.W. Pollak and G. Yassin are with the Department of Physics (Astrophysics), University of Oxford, Oxford, U.K. (e-mail: john.garrett@physics.ox.ac.uk).

M. Henry is with RAL Space, Science and Technology Facilities Council, UKRI, Oxfordshire, U.K.

Manuscript received March 7, 2019; revised June 25, 2019.

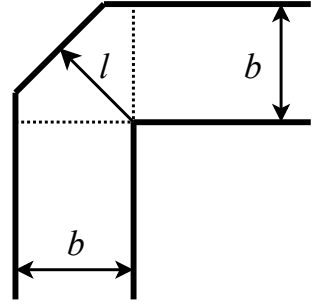

(a)

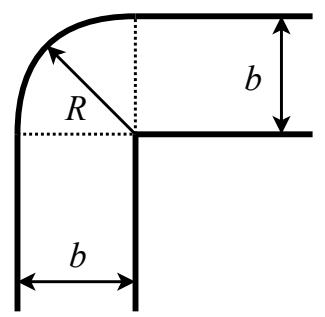

(c)

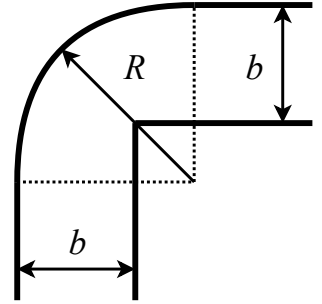

(b)

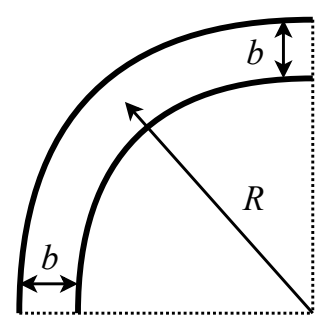

(d)
Fig. 1. Cross-sections of common E-plane waveguide bends: (a) mitered bends, (b,c) rounded bends, and (d) smooth arcs. For mitered bends, the optimal $l / b$ ratio is 0.86 [4], and for rounded bends, the optimal $R / b$ ratio is 1.328 [5]. The rounded bend in (c) uses $R / b=1$, which allows it to be machined using a cutting tool with diameter $b$.

very precise fabrication using computer numerically controlled (CNC) milling machines and they result in large waveguide circuits due to the large radii of the bends. This can be troublesome at millimeter wavelengths, both because of the small size of the components and the high conduction loss of the waveguides.

Here we present a new technique to create high-frequency E-plane waveguide bends. This technique was designed to (a) use split-block fabrication, (b) use a cutting tool that has a diameter equal to the waveguide's height, (c) use only linear channels in order to not require a CNC milling machine, and (d) create a compact $90^{\circ}$ bend that minimizes the size and the insertion loss of the waveguide circuit. This new design will be beneficial for creating high-frequency waveguide circuits that are both cost effective and low loss, such as those needed for radio astronomy, vehicular radar and next generation telecommunication systems.

\section{DESIGN}

The design of the new E-plane waveguide bend is shown in Fig. 2. It consists of two linear channels with a notch left in the outside corner. This design is intended for split-block fabrication and it assumes that the milling machine's cutting 


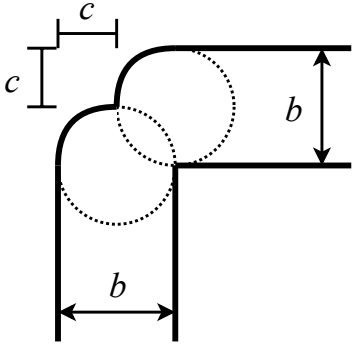

Fig. 2. The design of the new E-plane split-block waveguide bend. The circles represent the size of the cutting tool, which has a diameter equal to the waveguide's height, $b$. The dimension $c$ controls the size of the notch.

tool has a diameter equal to the waveguide's height. The notch in the outside corner is created by stopping the cutting tool before it fully reaches the bend. The position where the cutting tool stops is labeled as dimension $c$ in Fig. 2.

The new design was first scaled to the WR5.1 waveguide size (inner dimensions: $1.30 \times 0.65 \mathrm{~mm}$ ) and simulated using full-wave electromagnetic simulation software (ANSYS High Frequency Structural Simulator, Release 15.0; HFSS). The dimension $c$ was then optimized by minimizing the reflection coefficient over the operational bandwidth of the waveguide (140-220 GHz). Through these simulations, the optimal $c / b$ ratio was found to be $c / b=0.426$, which provides a reflection coefficient below $-28 \mathrm{~dB}$ across the entire frequency range. As long as the dimension $c$ is within $\pm 5 \%$ of the optimal value, the reflection coefficient is always below $-20 \mathrm{~dB}$ (although, typically much lower).

As seen in Fig. 3, the new design provides similar results to the mitered and rounded waveguide bends. However, the advantage of the new design is that it can be fabricated with a cutting tool that has a diameter equal to the waveguide's height, which is an important consideration at millimeter wavelengths. The performance is not as good as the smooth arc, but the new notch design is more compact, it can be machined using only linear channels (making it easier to fabricate without computer control), and it provides acceptable performance for most applications. Furthermore, because this bend does not require a computer-controlled milling machine, it can be very useful for making repairs to waveguide circuits, as discussed in [6].

\section{Physical Prototypes}

In order to validate the simulated results, a physical prototype of the waveguide bend was created for the Ku-band (12-18 GHz). This bend was designed using a WR62 waveguide (inner dimensions: $15.8 \times 7.9 \mathrm{~mm}$ ), so the dimension $c$ was set to $3.37 \mathrm{~mm}$, which is $42.6 \%$ of the waveguide's height. The waveguide bend was then machined in aluminum using a non-CNC milling machine.

The finished Ku-band prototype was tested on an Anritsu 37369C vector network analyzer (VNA) that was calibrated using the Short-Short-Load-Thru (SSLT) technique. The measured results are compared to simulated results from HFSS in Fig. 4. Note that the simulation was conducted once assuming perfect electric conductor (PEC) surfaces and once

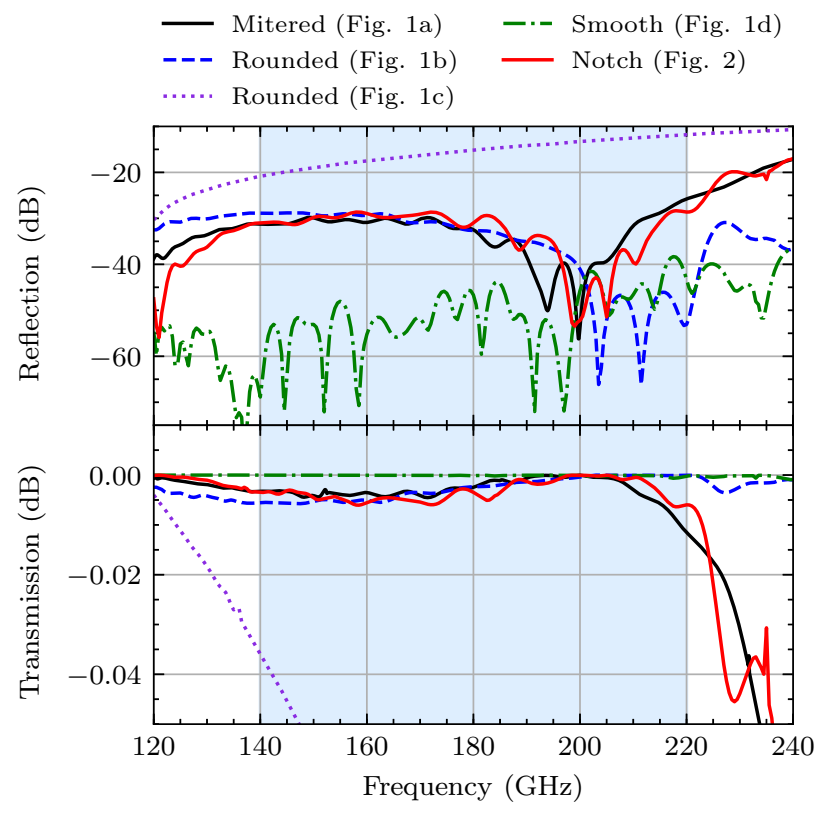

Fig. 3. Simulated performance of the waveguide bends from Fig. 1 and Fig. 2. This simulation used a WR5.1 waveguide size. The pale blue region represents the frequency range of the waveguide $(140-220 \mathrm{GHz})$. The radius of the smooth arc was set to $2 \lambda_{g}$ where $\lambda_{g}$ is the guided wavelength.

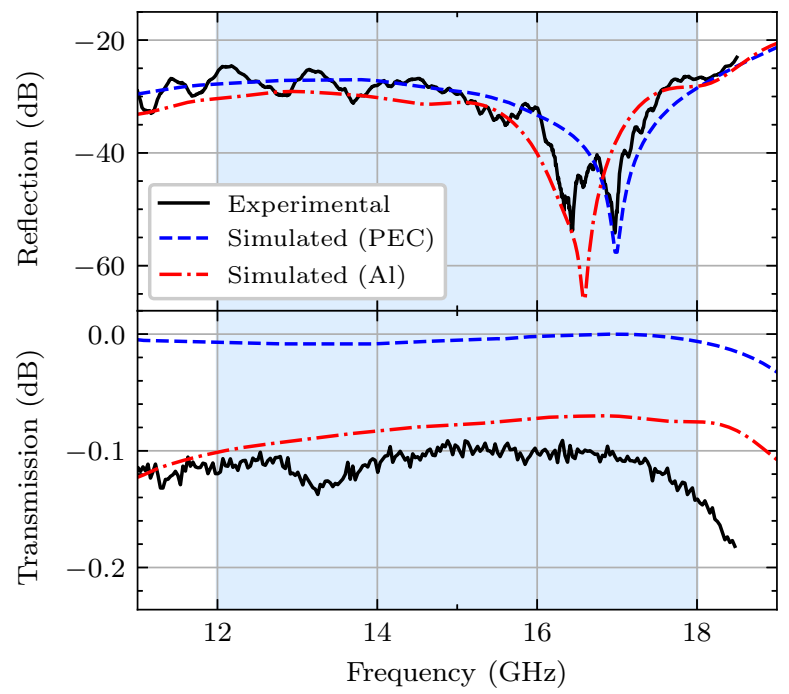

Fig. 4. Comparison between measured and simulated results of a WR62 waveguide bend using the new notch design. For "Simulated (PEC)", the waveguide walls were assumed to be perfect electric conductors (PEC), and for "Simulated (Al)", the conductivity of the waveguide walls was set to that of aluminum $\left(3.8 \times 10^{7} \mathrm{~S} / \mathrm{m}\right)$ and the surface roughness was set to $1.5 \mu \mathrm{m}$. In all cases, the total length of the waveguide was $20 \mathrm{~cm}$.

assuming the properties of aluminum (i.e., a conductivity of $3.8 \times 10^{7} \mathrm{~S} / \mathrm{m}$ and a surface roughness of $1.5 \mu \mathrm{m}$ ).

Fig. 4 shows good agreement between the experimental and simulated reflection results. The experimental transmission results are slightly lower than the simulated results, but the difference is less than $0.07 \mathrm{~dB}$ across the entire band. By comparing the simulation using PEC surfaces to the simulation using aluminum surfaces, we estimate that most of the loss in 


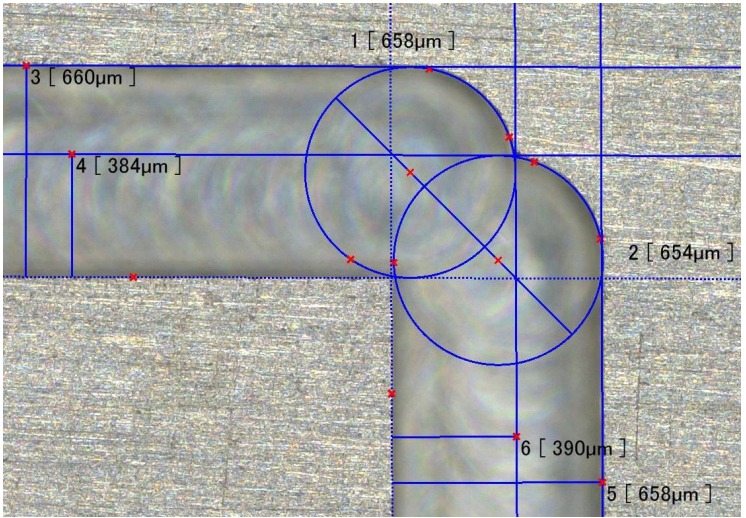

Fig. 5. An image of the WR5.1 waveguide bend taken with a Keyence VHX700F microscope. The dimensions were measured using the Keyence software. Dimensions \#1/\#2 measure the diameter of the cutting tool, \#3/\#5 measure the waveguide height, and \#4/\#6 measure the dimension $c$ from Fig. 2.

the experimental system was due to conduction losses in the waveguide walls, and not due to the bend itself.

To confirm that this technique is also feasible at millimeter wavelengths, another physical model was fabricated in the WR5.1 waveguide size. A magnified image of this bend is shown in Fig. 5. The dimension $c$ for this waveguide size should be $276 \mu \mathrm{m}$ and the waveguide height should be $648 \mu \mathrm{m}$. As seen in Fig. 5, the measured waveguide heights are $660 \mu \mathrm{m}$ and $658 \mu \mathrm{m}$, and the measured offsets are $276 \mu \mathrm{m}$ and $268 \mu \mathrm{m}$, so the physical model is within $2 \%$ of the intended waveguide height and within $3 \%$ of the intended offset. This prototype used two $4 \mathrm{~mm}$ diameter dowel pins to align the split-blocks, and standard UG-387 waveguide flanges.

The physical model was tested on a Keysight VNA with WR5.1 frequency extenders from Virginia Diodes, Inc. Very good agreement was found between the experimental and simulated transmission results, specifically the "Simulated (Al)" results, as seen in Fig. 6. Similar to the results from the WR62 waveguide (Fig. 4), the difference between the simulation using PEC waveguide walls and the simulation using aluminum waveguide walls suggests that nearly all of the insertion loss seen in the experimental system was due to conduction losses in the waveguide walls. Better transmission results would be possible if the waveguide bend were made from a more conductive metal, such as cooper, or if it were operated at a lower temperature, as is the case when the bend is used in astronomical receivers.

The reflection results are also shown in Fig. 6. The experimental results were time-gated to isolate the reflections from the waveguide bend, but they are still significantly higher than the simulated results from HFSS. We attribute this difference to artifacts from the time-gating procedure because (1) the reflections from the waveguide flanges were very strong, (2) the reflection from the bend was very weak, and (3) the reflections were difficult to separate in the time-domain. A longer waveguide before the bend or a wider frequency sweep would allow us to separate the two reflections with more accuracy. It is also possible that these results were affected by calibration drift in the VNA. Regardless, the new waveguide bend provides a reflection coefficient below $-23 \mathrm{~dB}$ across

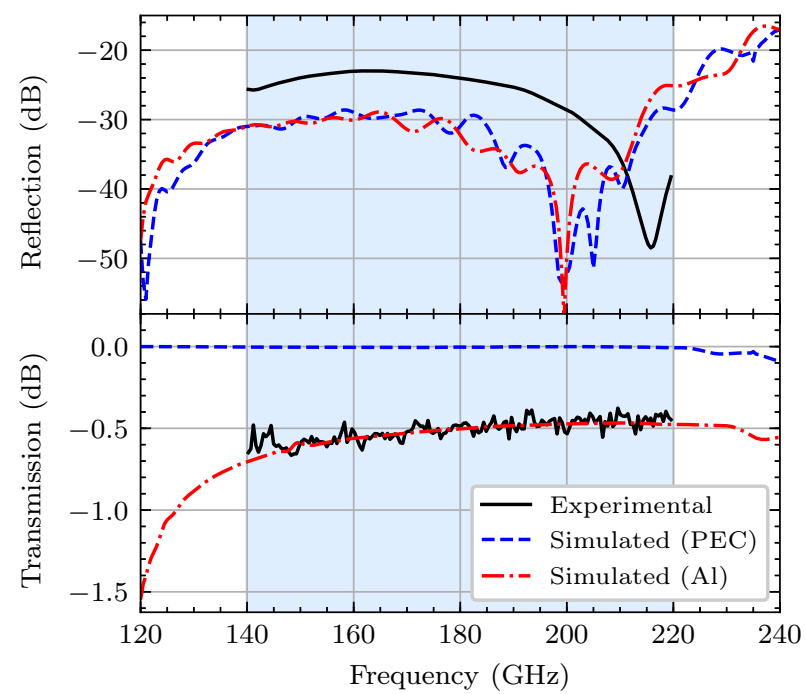

Fig. 6. Comparison between measured and simulated results of a WR5.1 waveguide bend using the new notch design. Similar to Fig. 4, "Simulated (PEC)" used PEC waveguide walls and "Simulated (Al)" used aluminum waveguide walls (with a surface roughness of $0.3 \mu \mathrm{m}$ ). For each case, the total length of the waveguide was $3 \mathrm{~cm}$. In the time-domain, the experimental reflection results show three peaks: one from the input waveguide flange, one from the bend, and one from the output waveguide flange. The reflection from the bend was isolated by time-gating the center peak (using a Kaiser window with center: $0.142 \mathrm{~ns}$, span: $0.21 \mathrm{~ns}$ ).

the entire operational bandwidth, which is acceptable for most applications at millimeter wavelengths.

\section{CONCLUSION}

We have presented a new high-frequency E-plane waveguide bend for split-block fabrication. This design can be created on a non-CNC milling machine using only linear passes with a cutting tool that has a diameter equal to the waveguide's height. Simulations of this new bend have shown a reflection coefficient below $-28 \mathrm{~dB}$ across the entire operation bandwidth of a WR5.1 waveguide. Physical models in the WR62 and WR5.1 waveguide sizes have validated these simulations and shown that this technique is feasible at millimeter wavelengths. This design will help to produce both cost effective and compact waveguide circuits, supporting new millimeterwave technology and applications.

\section{REFERENCES}

[1] H. Yang, Y. Dhayalan, X. Shang, M. J. Lancaster, B. Liu, H. Wang, M. Henry, and P. G. Huggard, "WR-3 Waveguide Bandpass Filters Fabricated Using High Precision CNC Machining and SU-8 Photoresist Technology," IEEE Trans. Terahertz Sci. Technol., vol. 8, no. 1, pp. 100107, Jan. 2018.

[2] A. R. Kerr, "Elements for E-Plane Split-Block Waveguide Circuits," ALMA Partnership, Tech. Rep. 381, 2001.

[3] A. R. Kerr and N. Horner, "A Split-Block Waveguide Directional Coupler," ALMA Partnership, Tech. Rep. 432, 2002.

[4] A. Casanueva, J. A. Pereda, and A. Mediavilla, "Optimum Compact Hand E-plane Corners in Rectangular Waveguide," Microw. Opt. Technol. Lett., vol. 42, no. 6, pp. 494-497, Jul. 2004.

[5] A. Abramowicz, "Nearly perfect right angle bends," in MIKON 2008, 17th International Conference on Microwaves, Radar and Wireless Communications, Warsaw, Poland, May 2008.

[6] J. D. Garrett, "A 230 GHz Focal Plane Array Using a Wide IF Bandwidth SIS Receiver,” DPhil thesis, University of Oxford, 2018. 